\title{
Effective transition rates for epitaxial growth using fast modulation
}

\author{
Martha A. Gallivan* \\ School of Chemical and Biomolecular Engineering, Georgia Institute of Technology, Atlanta, Georgia 30332, USA \\ David G. Goodwin and Richard M. Murray \\ Division of Engineering and Applied Science, California Institute of Technology, Pasadena, California 91125, USA
}

(Received 31 December 2003; published 13 July 2004)

\begin{abstract}
Thin-film deposition is an industrially important process that is highly dependent on the processing conditions. Most films are grown under constant conditions, but a few studies show that modified properties may be obtained with periodic inputs. However, assessing the effects of modulation experimentally becomes impractical with increasing material complexity. Here we consider periodic conditions in which the period is short relative to the time scales of growth. We analyze a stochastic model of thin-film growth, computing effective transition rates associated with rapid periodic process parameters. Combinations of effective rates may exist that are not attainable under steady conditions, potentially enabling new film properties. An algorithm is presented to construct the periodic input for a desired set of effective transition rates. These ideas are demonstrated in three simple examples using kinetic Monte Carlo simulations of epitaxial growth.
\end{abstract}

DOI: 10.1103/PhysRevB.70.045409

PACS number(s): 68.90.+g, 81.15.- z

\section{INTRODUCTION}

Thin-film deposition is a critical step in the manufacture of many devices, including integrated circuits and microelectromechanical systems. This often-complex process is highly dependent on process conditions such as temperature and pressure. Process development for a new material system has historically been accomplished empirically, but as materials become more complex, this approach becomes increasingly difficult. Even when the process parameters are held constant throughout deposition, the number of experiments required to probe the parameter space grows exponentially with the number of parameters (in the case of a multispecies film, the process conditions might include gas concentration for each species, plus substrate temperature, ion bombardment rate, and plasma power). Exploring the effects of time-varying process conditions quickly becomes impractical, in either experiment or simulation.

Conditions for thin-film deposition are often held constant, unless the process is inherently periodic, as is pulsed laser deposition. The morphology of films deposited by pulsed layer deposition and molecular-beam epitaxy have been compared in simulation by Taylor and Atwater ${ }^{1}$ to isolate the effects of a time-varying process. Deliberate modulation of process conditions has also been investigated. ${ }^{2-7}$ However, even in the case of a simple single-species system, the effect of flux modulation on film properties such as surface roughness is not completely clear. Compared to a film grown under constant flux with the same mean growth rate, arguments can be made that films grown with modulated flux should be rougher ${ }^{1}$ or smoother. ${ }^{4}$

When considering periodic variation of the process conditions, two questions are of interest. First, under what conditions do films grown with periodic variation of process parameters differ from those grown under steady conditions with the same mean process conditions? Presumably, the effects will depend on the magnitude and frequency of the variation, and will depend on the relative phase of different inputs. The second question is whether or not the same effects are achievable by other steady process conditions. If not, then it may be possible to grow films with modulation that have properties that differ from those achievable with any set of steady conditions.

Periodic growth strategies have been exploited to alter the ratio of various surface events. Olson et al. ${ }^{8}$ modulated the precursor flux during diamond growth to suppress gas-phase reactions, preventing the production and subsequent adsorption of hydrocarbons. Sugiyama, Matsura, and Murota ${ }^{9}$ instead alternated between precursor flux and plasma power to prevent the production and adsorption of chlorine radicals during etching. Periodic conditions have also been utilized in electrodeposition by Svensson, Wahlstrom, and Holmbom ${ }^{10}$ to obtain altered film stoichiometry through current modulation.

In this paper we show formally how the ratio of surface events can be altered with periodic growth conditions. In particular, we focus on modulation that is fast relative to the time scales of growth. In this limit, one would not expect the film to rapidly respond to each individual pulse, but instead to change only slightly over each modulation period. We construct a framework from which to view fast modulation, by deriving effective rates for the surface events. These rates may or may not be attainable with steady conditions, depending on the functional form of the transition rates relative to the process conditions.

\section{LATTICE MODEL OF EPITAXIAL GROWTH}

Thin-film growth encompasses a wide range of length and time scales, with corresponding models at each scale, from quantum mechanics to continuum theory. We require a model which accurately captures the atomic-scale effects of the process conditions, and which describes film properties of interest on the time scales of film growth. A lattice model for crystal growth provides a good balance between these re- 


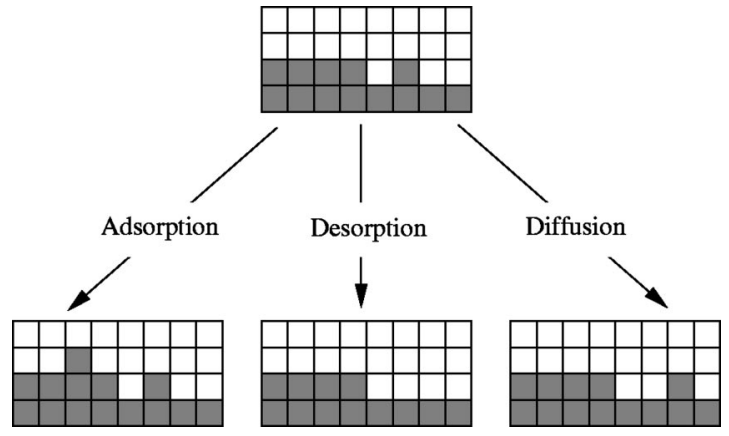

FIG. 1. Illustration of three common transition mechanisms for a two-dimensional lattice associated with a one-dimensional substrate.

quirements. The lattice model is described mathematically by a high-dimensional probabilistic master equation, but is typically simulated using a stochastic Monte Carlo method. ${ }^{11}$

The two key components of the lattice model are the rigid lattice to which atoms are constrained and the mechanisms and rates of atomic transitions between points on the lattice. The lattice represents the underlying crystal structure of the material, and is assumed to be fixed. The transition mechanisms are defined in terms of lattice configurations, in which unique configurations are distinguished by differences in the occupancy of the lattice sites. Each unique transition mechanism is associated with a set of configuration pairs, in which the first element of each pair may transition into the second element. Three common transition mechanisms-adsorption, desorption, and diffusion-are illustrated in Fig. 1. The dependence on the process conditions enters through the rates of transition associated with each transition mechanism.

\section{A. Master equation}

A master equation describes the evolution in time of the probabilities of each lattice configuration. ${ }^{12}$ Let the symbol $H$ denote a particular configuration, and $k^{H_{a} \rightarrow H_{b}}$ the transition rate from $H_{a}$ to $H_{b}$. Figure 2 illustrates two of the many possible configurations for a one-dimensional substrate. In the figure, a transition mechanism from $H_{a}$ to $H_{b}$ is the adsorption of an atom at the third site from the left. A transition

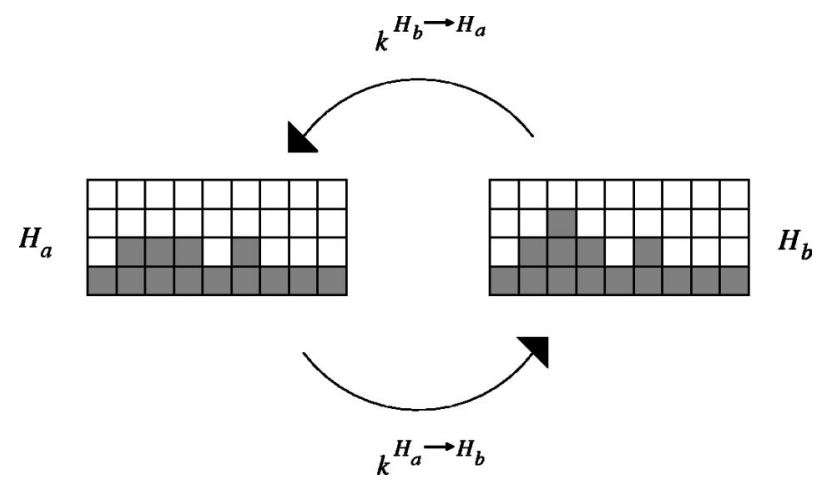

FIG. 2. Two possible configurations of a two-dimensional lattice associated with a one-dimensional substrate, with corresponding transitions and transition rates. from $H_{b}$ to $H_{a}$ may occur through desorption at this site.

The number of configurations grows exponentially with the size of the lattice. For a substrate of $100 \times 100$ sites and a maximum height of only 10 , the number of configurations is $10^{100 \times 100}$. Although this number can be reduced by physical assumptions and symmetry arguments, it is clearly impossible to directly simulate the master equation for any realistic domain size. We use the structure of the master equation in our analysis, but continue to perform stochastic simulations with the kinetic Monte Carlo method.

The master equation may be expressed in terms of sums over all the configurations

$$
\frac{d}{d t} P_{H}(t)=\sum_{H^{\prime}} k^{H^{\prime} \rightarrow H} P_{H^{\prime}}(t)-\sum_{H^{\prime}} k^{H \rightarrow H^{\prime}} P_{H}(t),
$$

where $t$ is time and $P_{H}(t)$ is the time-dependent probability of configuration $H$. The first term on the right-hand side reflects transitions from other configurations $H^{\prime}$ into configuration $H$, and the second term represents transitions out of configuration $H$. Any expected property, such as island density or root-mean-square roughness, may be expressed as a linear combination of the probabilities:

$$
\langle Y\rangle(t)=\sum_{H} P_{H}(t) Y(H),
$$

where $Y$ is the quantity of interest, $Y(H)$ is the value of $Y$ associated with configuration $H$, and $\langle Y\rangle(t)$ denotes the time dependent expected value of $Y$.

Although there are a large number of configurations and possible transitions between configurations, $k^{H_{a} \rightarrow H_{b}}$ may only take $m$ distinct values associated with the $m$ unique transition mechanisms, or zero if no allowable transition between two configurations exists. The master equation may be recast as a sum over the $m$ transition mechanisms. We use the symbol $k_{i}^{H \rightarrow H^{\prime}}$ to denote the transition rate associated with a particular transition mechanism, and express the master equation as

$$
\frac{d}{d t} P_{H}(t)=\sum_{i=1}^{m}\left(\sum_{H^{\prime}} k_{i}^{H^{\prime} \rightarrow H} P_{H^{\prime}}(t)-\sum_{H^{\prime}} k_{i}^{H \rightarrow H^{\prime}} P_{H}(t)\right) .
$$

Taking $k_{i}$ to be the rate of transition through mechanism $i$, note that $k_{i}^{H \rightarrow H^{\prime}}$ may take only two values: zero, if no transition from $H$ to $H^{\prime}$ is allowed via mechanism $i$, or $k_{i}$, if a transition through mechanism $i$ is allowed.

We next rewrite Eq. (3) as a vector equation for the probability vector $\mathbf{P}$

$$
\frac{d}{d t} \mathbf{P}=\sum_{i=1}^{m}\left(k_{i} N_{i}^{\text {in }} \mathbf{P}-k_{i} N_{i}^{\text {out }} \mathbf{P}\right),
$$

where $N_{i}^{\text {in }}$ and $N_{i}^{\text {out }}$ are matrices that represent the allowable transitions either into or out of a configuration through mechanism $i . N_{i}^{\text {in }}$ and $N_{i}^{\text {out }}$ contain mostly zero elements, since a transition mechanism will likely not exist between two randomly selected configurations. As the last step we combine $N_{i}^{\text {in }}$ and $N_{i}^{\text {out }}$ into a single matrix $N_{i}=N_{i}^{\text {in }}-N_{i}^{\text {out }}$ to arrive at our final form of the master equation 


$$
\frac{d}{d t} \mathbf{P}=\sum_{i=1}^{m} k_{i}(\mathbf{u}) N_{i} \mathbf{P}
$$

Equation (5) is equivalent to Eq. (1) —only the notation is different. We define $\mathbf{u}$ to be the vector of process parameters and explicitly state the dependence of $k_{i}$ on $\mathbf{u}$ because it will be important in the analysis that follows.

\section{B. Kinetic Monte Carlo simulations}

The lattice model captures many key features of thin-film growth and has been used extensively over the past 30 years as the basis for Monte Carlo simulations. ${ }^{11,13}$ We perform kinetic Monte Carlo (KMC) simulations in this paper, using the formalism of Bortz ${ }^{14}$ to capture the correct evolution in time. Kinetic Monte Carlo simulations provide useful predictions of thin-film growth, but the rule-based simulations are not conducive to mathematical analysis. For example, the simulations are not invertible; the evolution of a film grown under particular process conditions can be computed, but the process parameters required to achieve a film with particular film properties cannot.

The analysis developed in the remainder of the paper applies generally to any lattice model. However, throughout the paper we demonstrate the analysis on specific model systems through KMC simulations. These simulations are based on a cubic lattice in which each atom has a maximum of six nearest neighbors - one on each side for a total of four, one above, and one below. Additionally, we make the solid-onsolid approximation, in which no vacancies in the crystal are permitted; equivalently, every atom must have a neighbor below it. Periodic boundaries are utilized to simulate an infinite surface, since an actual film is much larger than the simulation domain. A simulation domain of $300 \times 300$ was used, with selected simulations also computed on 200 $\times 200$ and $400 \times 400$ domains.

\section{DERIVATION OF EFFECTIVE TRANSITION RATES}

We now analyze the effects of periodic process conditions for the particular case when the process condition period is short relative to the time scales of film growth. In this situation the film is not able to respond fast enough to keep up with changes in the process parameters. Instead, the film evolves as if the transition rates were replaced by constant "effective" transition rates. We employ the method of averaging ${ }^{15}$ to compute these effective transition rates. Similar application of the averaging theorem has been applied to mechanical systems with periodic inputs. ${ }^{16}$

The method of averaging may be applied to a differential equation of the form

$$
\frac{d}{d t} \mathbf{x}=\epsilon f(\mathbf{x}, t),
$$

where $\mathbf{x}$ is a vector of dimension $n, \epsilon$ is a constant, and $f$ is a function with continuous first and second derivatives. When $f$ is periodic in $t$ with period $\tau$, such that $f(t+\tau)$ $=f(t)$, its average is defined as

$$
\bar{f}(\hat{\mathbf{x}}) \equiv \frac{1}{\tau} \int_{0}^{\tau} f(\hat{\mathbf{x}}, t) d t,
$$

and the averaged equation is defined as

$$
\frac{d}{d t} \hat{\mathbf{x}}=\epsilon \bar{f}(\hat{\mathbf{x}})
$$

where $\hat{\mathbf{x}}$ is a vector of the same dimension as $\mathbf{x}$. The averaging theorem relates the difference between $\mathbf{x}$ and $\hat{\mathbf{x}}$ to the size of $\epsilon$. Specifically,

$$
|\mathbf{x}(t)-\hat{\mathbf{x}}(t)|=O(\epsilon)
$$

on a time scale of $O(C / \epsilon)$ if $\left|\mathbf{x}\left(t_{0}\right)-\hat{\mathbf{x}}\left(t_{0}\right)\right|<O(\epsilon)$ for some initial time $t_{0}$. The constant $C$ is independent of $\epsilon$. Notice that when $\epsilon$ is small, the averaged equation (8) is a good approximation for the original equation (6). Refer to Wiggins ${ }^{15}$ for further discussion and a proof of the averaging theorem.

The averaging theorem may be applied to the master equation (3) when the process parameters are periodic, i.e. $\mathbf{u}=\mathbf{u}(\omega t)$ with frequency $\omega=2 \pi / \tau$. Restating Eq. (3) to emphasize the dependence on time, we obtain

$$
\frac{d}{d t} \mathbf{P}=\sum_{i=1}^{m} k_{i}[\mathbf{u}(\omega t)] N_{i} \mathbf{P} .
$$

Before applying the method of averaging, Eq. (10) must be in the form of Eq. (6) with a small parameter $\epsilon$. With this goal we rescale time by $\omega$. Defining a new time $s \equiv \omega t$ and $\epsilon \equiv 1 / \omega$, Eq. (10) becomes

$$
\frac{d}{d s} \mathbf{P}=\epsilon \sum_{i=1}^{m} k_{i}[\mathbf{u}(s)] N_{i} \mathbf{P} .
$$

Setting $\mathbf{x}=\mathbf{P}$ and $f(\mathbf{x}, s)=\sum_{i=1}^{m} k_{i}[\mathbf{u}(s)] N_{i} \mathbf{x}$, and observing that the period in scaled time is $2 \pi$, the averaged version of Eq. (11) is

$$
\frac{d}{d s} \hat{\mathbf{P}}=\epsilon \sum_{i=1}^{m}\left(\frac{1}{2 \pi} \int_{0}^{2 \pi} k_{i}[\mathbf{u}(s)] d s\right) N_{i} \hat{\mathbf{P}} .
$$

We may express this more compactly by defining an "effective" transition rate $k_{\mathrm{eff}, i}$ as

$$
k_{\mathrm{eff}, i} \equiv \frac{1}{\tau} \int_{0}^{\tau} k_{i}[\mathbf{u}(\omega t)] d t=\frac{1}{2 \pi} \int_{0}^{2 \pi} k_{i}[\mathbf{u}(s)] d s .
$$

An effective transition rate is simply the average value of the transition rate over a period, and is not a function of time. In physical time $t$ the averaged version of Eq. (10) is then

$$
\frac{d}{d t} \hat{\mathbf{P}}=\sum_{i=1}^{m} k_{\mathrm{eff}, i} N_{i} \hat{\mathbf{P}} .
$$

The time scale on which the approximation is valid is $O(C / \epsilon)$ in scaled time $s$ but is $O(C)$ in physical time $t$, independent of the frequency. We explore the time scales of applicability further in the simulations of Sec. IV.

Notice that the averaged master equation (14) has the same form as Eq. (10); the transition rates $k_{i}$ are simply 
replaced by $k_{\mathrm{eff}, i}$. Application of fast periodic process conditions is equivalent to a film growth process with constant effective transition rates $k_{\text {eff }, i}$. If these effective rates are not attainable with constant process conditions, then altered film evolution may be possible. In practice, the process parameters are bounded, so we let $\mathbf{u}_{\min }$ and $\mathbf{u}_{\max }$ be the minimum and maximum values of $\mathbf{u}$, and ask the question: do there exist effective transition rates attainable with periodic process conditions in the range $\left[\mathbf{u}_{\min }, \mathbf{u}_{\max }\right]$ that are not attainable with constant parameters in $\left[\mathbf{u}_{\min }, \mathbf{u}_{\max }\right]$ ?

No single transition rate $k_{\text {eff }, i}$ can be outside the set of the $k_{i}$ 's attainable with constant parameters, since the effective rate is simply the time average over the instantaneous rate. However, new combinations of effective transition rates might be obtained. The ratio of the transition rates of various mechanisms strongly affects the evolution and final properties of a film-for example, the ratio of flux to surface diffusion is a key parameter in the evolution of island density and surface roughness. ${ }^{17,18}$

\section{EXAMPLES}

To demonstrate the idea of effective transition rates, we first consider a single-species system. The focus of this example is to show that the set of achievable effective rates depends on the relationship between the process inputs and the transition rates. A second example is then considered, which is motivated by chemical vapor deposition. When multiple species are delivered by a single precursor, temperature modulation can be used to alter a film's stoichiometry.

Both examples are based on a cubic lattice, have periodic boundary conditions, and disallow vacancies in the crystal. The two process parameters are the flux $F$ of precursors to the surface and the surface temperature $T$, so that $\mathbf{u}=\{F, T\}$. We assume that we have complete control over the flux and temperature within preset upper and lower bounds, such that $\mathbf{u}$ may be constant or a periodic function of time. In all cases the flux ranges between 0 and 1 monolayers/s, the temperature ranges between 400 and $600 \mathrm{~K}$, and the activation energies lie between 0.5 and $1.5 \mathrm{eV}$. However, in this study we use dimensionless quantities to further generalize the results. The time scale is taken from the maximum flux $F_{\max }$ and the energy scale is $k_{b} T_{\max }$, where $k_{b}$ is Boltzmann's constant and $T_{\max }$ is the upper bound on temperature. The length scale is set by the lattice spacing $a$. A dimensionless quantity will be denoted by an asterisk in the remainder of this section-for example, transition rate $k_{i}^{*}=k_{i} F_{\max }$, activation energy $E_{j}^{*}$ $=E_{j} / k_{b} T_{\max }$, and height $h^{*}=h / a$.

\section{A. Single-species example}

Three types of transitions are allowed in the singlespecies model: (1) adsorption of an atom from the gas onto the surface, (2) diffusion of an atom with no side neighbors along the surface, and (3) loss of an atom with no side neighbors to the gas. An atom with one or more side neighbors may undergo no transition and thus is permanently incorporated into the film. Two models for the transition rates are considered, and are given in Table I. The first transition
TABLE I. Transition rates for models 1 and 2. The dimensionless transition rates $k_{i}^{*}$ are functions of the dimensionless process parameters $F^{*}$ and $T^{*}$.

\begin{tabular}{cc}
\hline \hline Model 1 & Model 2 \\
\hline$k_{1}^{*}\left(F^{*}\right)=F^{*}$ & $k_{1}^{*}\left(F^{*}\right)=F^{*}$ \\
$k_{2}^{*}\left(T^{*}\right)=\nu_{2}^{*} \exp \left(-E_{2}^{*} / T^{*}\right)$ & $k_{2}^{*}\left(T^{*}\right)=\nu_{2}^{*} \exp \left(-E_{2}^{*} / T^{*}\right)$ \\
$k_{3}^{*}\left(T^{*}\right)=\nu_{3}^{*} \exp \left(-E_{3}^{*} / T^{*}\right)$ & $k_{3}^{*}\left(F^{*}, T^{*}\right)=F^{*} \nu_{3}^{*} \exp \left(-E_{3}^{*} / T^{*}\right)$ \\
\hline \hline
\end{tabular}

mechanism is adsorption; its rate is equal to the flux $F^{*}$ in both models (unity sticking coefficient). The second mechanism is surface diffusion. It is a thermally activated process with proportionality constant $v_{2}^{*}$ and activation energy $E_{2}^{*}$, and is again the same in both models. The only difference between models 1 and 2 is the transition rate for the third mechanism, which results in the removal of an atom from the surface. In the first model, the third mechanism is a thermally activated process, which is only dependent on the temperature, with a proportionality constant $v_{3}^{*}$ and activation energy $E_{3}^{*}$. However, in model 2 the third mechanism is an etching process, which is thermally activated as in model 1 but is also proportional to the flux. In model 1, atoms desorb at high temperature due to thermal effects, but in model 2 , high temperature and high flux must coincide to remove atoms from the surface.

First consider the evolution of a film described by model 1 , which we investigate through kinetic Monte Carlo simulations. The physical parameters are determined by selecting the values of the transition rates at the upper and lower bounds of the flux and temperature: $k_{1}^{*}\left(F_{\min }^{*}, T^{*}\right)=0$, $k_{2}^{*}\left(F^{*}, T_{\text {min }}^{*}\right)=0.01, k_{2}^{*}\left(F^{*}, T_{\text {max }}^{*}\right)=1000, k_{3}^{*}\left(F^{*}, T_{\text {min }}^{*}\right)=10$, and $k_{3}^{*}\left(F^{*}, T_{\max }^{*}\right)=1000$. As a final constraint we select $T_{\text {min }}^{*}$ $=2 / 3$ to obtain physically realistic activation energies.

Before analyzing periodic flux and temperature, we visualize the set of transition rates attainable with constant flux and temperature. Because there are only three transition mechanisms, we may plot the transition rates against each other and obtain the two-dimensional surface of transition rates. This surface is shown in Fig. 3. The surface is twodimensional because there are two process parameters and is bounded because the process parameters have upper and lower bounds.

Any combination of transition rates that is not on the surface of Fig. 3 cannot be obtained with constant flux and temperature. However, periodic inputs may produce a combination of effective transition rates that is not on the surface. Consider the input pictured in Fig. 4. The flux is set to the maximum value, but the temperature alternates between its upper and lower bounds. The set of effective rates associated with this periodic input may be computed with Eq. (13), and is denoted by the filled diamond in Fig. 3. These rates are not achievable with constant flux and temperature, and result in a decrease in desorption relative to diffusion and adsorption. An effective transition rate is the time average of the instantaneous rate over a period, and thus for the input in Fig. 4, the effective rate is the average of the transition rates at $\left\{F_{\text {max }}^{*}, T_{\max }^{*}\right\}$ and $\left\{F_{\max }^{*}, T_{\min }^{*}\right\}$. These instantaneous rates are marked by the open square and open triangle in Fig. 3. Any 


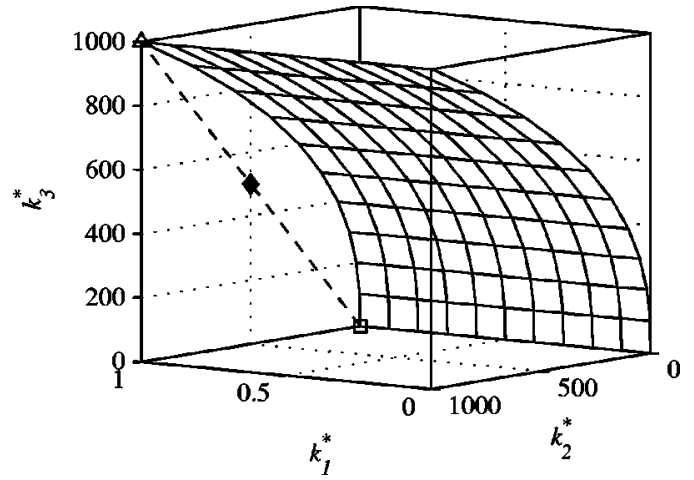

FIG. 3. The surface of transition rates achievable with constant process parameters for model 1 . The filled diamond marks the set of effective transition rates for the $50 \%$ duty cycle input in Fig. 4 . The instantaneous constant transition rates used to generate the set of effective rates are marked with the open square $\left(F^{*}=1\right.$ and $T^{*}$ $=2 / 3)$ and the open triangle $\left(F^{*}=1\right.$ and $\left.T^{*}=1\right)$.

point along the dashed line can be achieved by altering the duty cycle of the modulation of Fig. 4. Throughout this paper, open symbols are used for constant conditions, while filled symbols denote effective rates associated with modulated conditions.

Periodic process parameters enable new effective transition rates, which may ultimately result in altered film properties. We use kinetic Monte Carlo simulations to contrast the mean thickness $h^{*}$ and root-mean-square roughness $W^{*}$ obtained under constant and periodic inputs. Each simulation is run from $t^{*}=0$ to $t^{*}=100$, which results in a thickness of up to 100 layers. The mean of three simulations is shown in Fig. 5.

The simulations are performed for representative values of constant flux and temperature, as well as for the set of effective transition rates considered above. Notice that the final combination of thickness and roughness for the effective case could not be achieved by constant inputs in the fixed time interval we considered. The periodic input suppresses desorption relative to diffusion, enabling a thicker, smoother film. With constant flux and temperature we might be able to produce a film similar to that produced with the periodic inputs, but the growth time would be longer.

We will now analyze model 2, whose transition rates are shown in Table I. The physical parameters for Model 2 are selected by first setting $k_{1}^{*}\left(F_{\text {min }}^{*}, T^{*}\right)=0, k_{2}^{*}\left(F^{*}, T_{\max }^{*}\right)=1000$, and $k_{3}^{*}\left(F_{\text {max }}^{*}, T_{\text {max }}^{*}\right)=10000$. We also want $k_{2}^{*}\left(F^{*}, T_{\text {min }}^{*}\right)$ and $k_{3}^{*}\left(F_{\max }^{*}, T_{\min }^{*}\right)$ to be negligible, and consequently set $\nu_{2}^{*}$ $=10^{13}, \nu_{3}^{*}=10^{12}$, and $T_{\min }^{*}=2 / 3$.

Figure 6 contains the surface of transition rates achievable with constant inputs. Because the desorption rate is a function of both flux and temperature, the shape of the constant

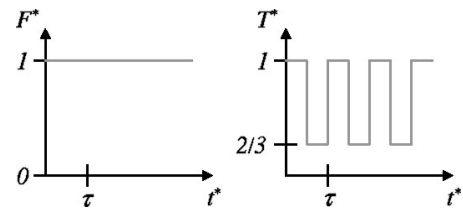

FIG. 4. A set of periodic process parameters for model 1 .

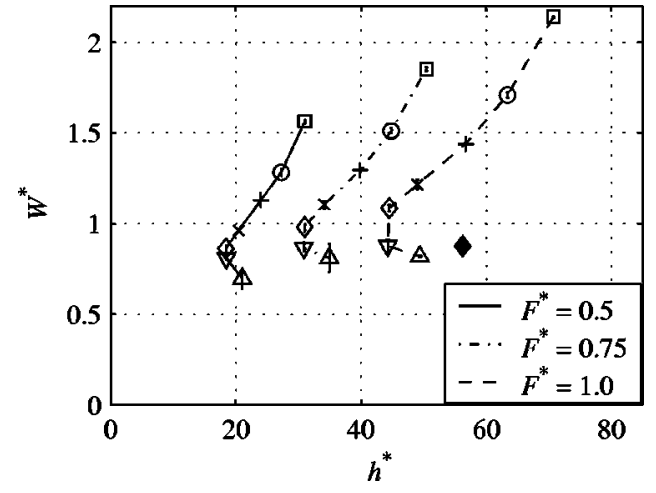

FIG. 5. KMC simulations of model 1: Final roughness $W^{*}$ vs thickness $h^{*}$ for various constant process parameters (open symbols) and for the set of effective transition rates (filled symbol). Each simulation is run up to $t^{*}=100$, and each symbol represents the mean of three simulations. Error bars denote the standard deviation, but are too small to be seen in most cases. Constant input simulations are marked by the open symbols, for $F^{*}=0.5,0.75$, and 1 ; and $T^{*}=2 / 3(\square), \quad 0.70(\bigcirc), \quad 0.73(+), \quad 0.78(\times), \quad 0.83(\diamond)$, $0.92(\nabla)$, and $1(\triangle)$. Effective rates associated with the modulated inputs of Fig. 4 are also simulated, as marked with the filled diamond $(\diamond)$.

input surface is qualitatively quite different from the surface associated with model 1 (Fig. 3). We again select a periodic input composed of two constant-parameter settings, this time alternating between high flux at low temperature, and low flux at high temperature, as pictured in Fig. 7. The rates associated with these two constant settings are marked with open symbols in Fig. 6; the filled diamond denotes the set of effective rates associated with the input in Fig. 7. Variations in the duty cycle of this input produce other effective rates, which lie along the dashed line in Fig. 6. The set of effective rates is dramatically different from any combination of rates achievable with constant inputs, which suggests that new film properties may also be obtained.

Kinetic Monte Carlo simulations of model 2 are shown in Fig. 8 for a range of constant parameters and for the effective

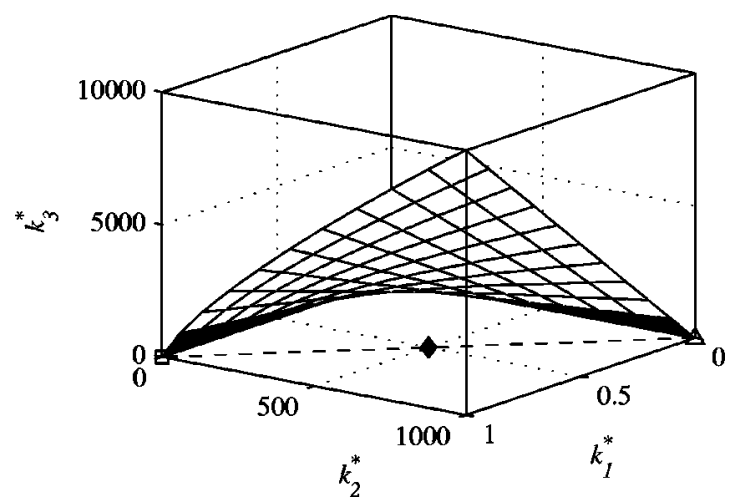

FIG. 6. The surface of transition rates achievable with constant process parameters for model 2 . The filled diamond marks the set of effective transition rates for the $50 \%$ duty cycle input in Fig. 7 . The instantaneous constant transition rates used to generate the set of effective rates are marked with the square $\left(F^{*}=1\right.$ and $\left.T^{*}=2 / 3\right)$ and the triangle $\left(F^{*}=0\right.$ and $\left.T^{*}=1\right)$. 


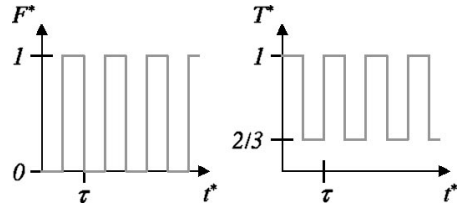

FIG. 7. A set of periodic process parameters for model 2 .

transition rates of Fig. 7. We again focus on the combinations of roughness and thickness that can be obtained at $t^{*}=100$. The effective rate surface is qualitatively different from the constant input surfaces-it lies almost completely outside the region defined by the constant inputs.

Figure 9 shows the final surfaces of a film grown under the set of effective transition rates and a much rougher film of a similar final thickness grown under the constant process parameters of $F^{*}=1.0$ and $T^{*}=0.78$. A significant fraction of former surface is covered by smooth terraces, while the latter is dominated by step edges. The physical mechanism for the smoothing associated with modulation is simple- under constant process conditions the temperature must be raised to induce smoothing, which results in the loss of atoms due to desorption. The use of periodic parameters enables the suppression of desorption, while still allowing smoothing through diffusion.

\section{B. Multispecies example}

We now consider a multispecies KMC simulation, which is motivated by a problem in chemical vapor deposition. In physical vapor deposition processes, one may be able to independently adjust the flux of each species to control the film composition, but in chemical vapor deposition, a single precursor may contain multiple film components. For example, in gallium arsenide, residual carbon from the gallium precur-

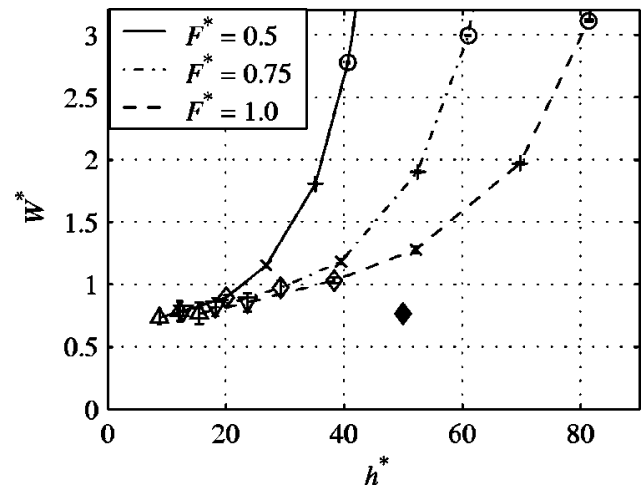

FIG. 8. KMC simulations of model 2: Final roughness $W^{*}$ vs thickness $h^{*}$ for various constant process parameters and for the set of effective transition rates. Each simulation is run up to $t^{*}=100$, and each symbol represents the mean of three simulations. Error bars denote the standard deviation, but are too small to be seen in most cases. Constant input simulations are marked by the open symbols, for $F^{*}=0.5,0.75$, and 1 ; and $T^{*}=0.70(\bigcirc), 0.73(+)$, $0.78(\times), 0.83(\diamond), 0.92(\nabla)$, and $1(\triangle)$. Effective rates associated with the modulated inputs of Fig. 4 are also simulated, as marked with the filled diamond $(\bullet)$.
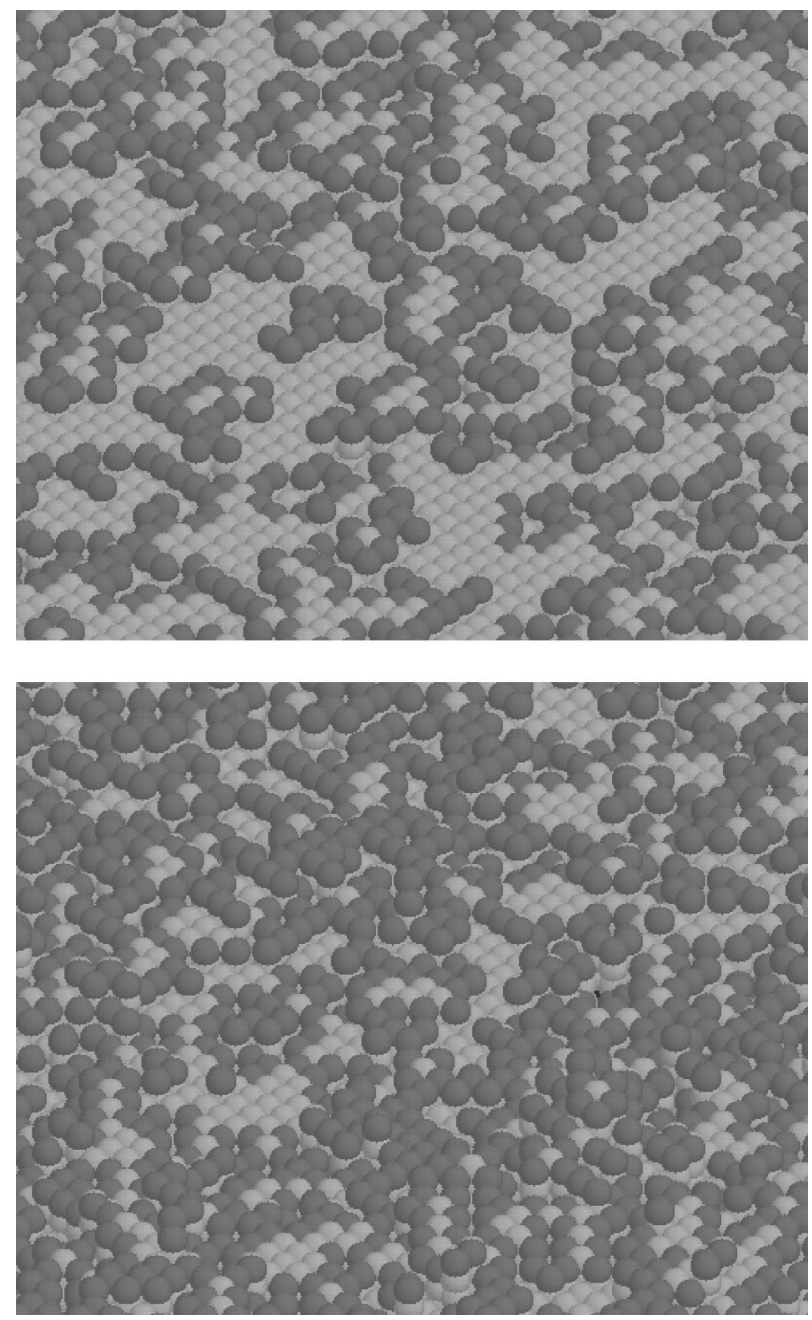

FIG. 9. KMC simulations of model 2. Only a portion of the $300 \times 300$ domain is shown. (a) Final surface of a film grown at the effective transition rates, marked by the filled diamond in Fig. 8. (b) Final surface of a film grown at the constant parameters $F^{*}=1.0$, $T^{*}=0.78$, marked by an $\times$ in Fig. 8 . Each atom with four side neighbors is light colored-atoms with at least one empty side bond are dark.

sor has been used as a dopant. ${ }^{19}$ In this situation the film composition is dependent on surface transitions that are strongly dependent on temperature. Activation energy models have been developed for gallium arsenide to quantify the dependence of precursor dissociation and desorption on temperature. ${ }^{20}$ The transition rates considered here are motivated by this model, in which the activation energy for precursor dissociation is less than the energy for dopant desorption, but greater than the energy for desorption of the precursor.

In particular, four events are considered: (1) adsorption, equal to the precursor flux $F$; (2) precursor desorption, with activation barrier $E_{2}^{*}=9.69$; (3) precursor dissociation, with $E_{3}^{*}=19.4$; and (4) dopant desorption, with $E_{4}^{*}=29.1$. Vibrational frequencies are set such that $k_{2}\left(T_{\max }\right)^{*}=10, k_{3}\left(T_{\max }^{*}\right)$ $=1000$, and $k_{4}\left(T_{\max }^{*}\right)=1000$, with $T_{\min }^{*}=2 / 3$. The corresponding rate "surface" is shown in Fig. 10, for $k_{2}, k_{3}$, and $k_{4}$. Each of these rates depends only on the temperature, resulting in a 

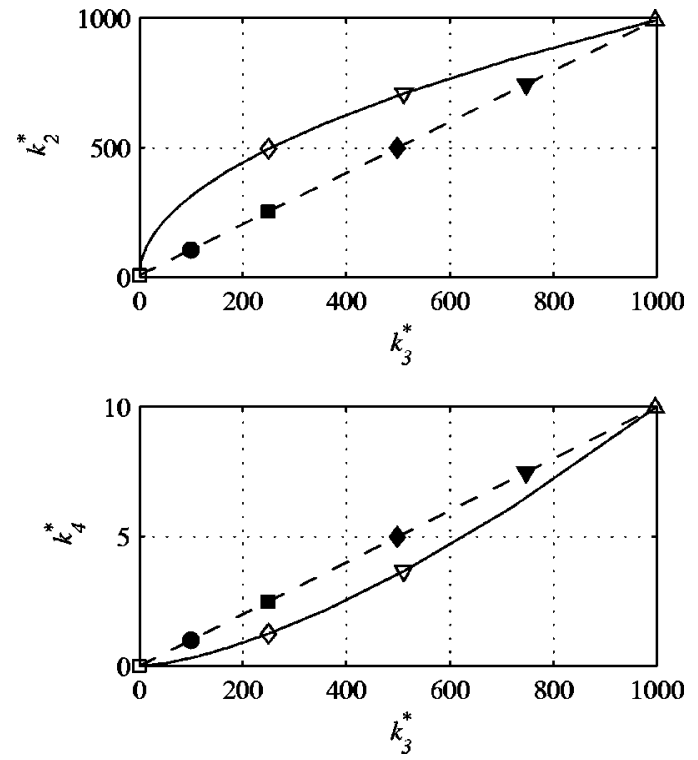

FIG. 10. Combinations of surface transition rates attainable with the multispecies model under constant temperature and flux (solid line). The $50 \%$ duty cycle modulation of Fig. 4 is marked $(\checkmark)$, along with other duty cycles with $25 \%(\boldsymbol{\nabla}), 75 \%(\boldsymbol{\square})$, and $90 \%(\bullet)$ of each period spent at $T_{\min }$. The instantaneous constant transition rates used to generate the effective rates are marked with the open square $\left(F^{*}=1\right.$ and $\left.T^{*}=2 / 3\right)$ and the open triangle $\left(F^{*}=1\right.$ and $T^{*}$ $=1)$. Additional constant inputs with $F^{*}=1$ and $T^{*}=0.93(\diamond)$ and $T^{*}=0.97(\nabla)$ are also shown.

one-dimensional curve of transition rates attainable with constant temperature. This curve is difficult to visualize on a three-dimensional plot, so two-dimensional plots are shown instead. Temperature modulation between $T_{\min }^{*}$ and $T_{\max }^{*}$ is represented by the dashed lines of Fig. 10 for the spectrum of possible duty cycles. Temperature modulation suppresses the effective rate for $k_{4}$ relative to $k_{3}$, and enhances $k_{2}$ relative to $k_{3}$.

The corresponding KMC simulations are shown in Fig. 11 , for growth up to $t^{*}=100$. Final thickness is plotted versus $d^{*}$, the total amount of secondary dopant species (measured in layers). As the constant growth temperature is increased from $T_{\min }^{*}$ to $T_{\max }^{*}$, the final thickness increases because the ratio of precursor dissociation to precursor desorption is enhanced. However, as the temperature is increased past $T^{*}$ $=0.93$ the dopant desorption becomes significant, leading to a reduction in $d^{*}$.

Temperature modulation leads to different combinations of thickness and composition. In particular, thickness is enhanced since the precursor desorption rate is suppressed relative to dissociation. The dopant desorption rate is raised slightly, which could potially lower the resulting value of $d^{*}$. However, this effect is more than compensated by the enhanced growth rate, in which dopant atoms on the surface are more rapidly incorporated into the bulk.

\section{CONSTRUCTION OF EFFECTIVE TRANSITION RATES}

\section{A. Modulation frequency for limiting behavior}

We derived effective transition rates associated with fast periodic process conditions, but have not yet addressed what

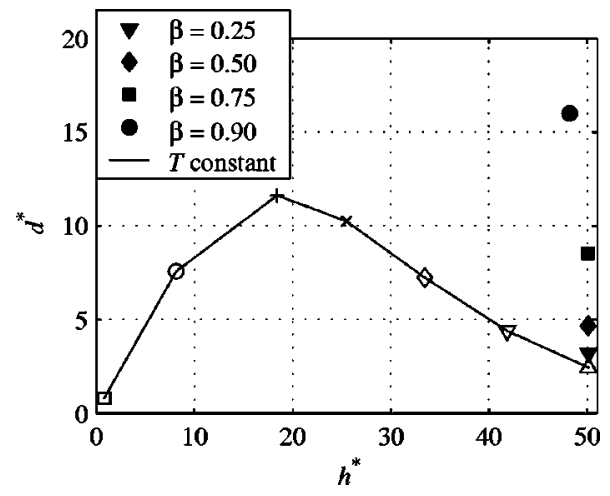

FIG. 11. KMC simulations of the multispecies model: Final dopant incorporation $d^{*}$ vs thickness $h^{*}$ for various constant process parameters and for the set of effective transition rates. Each simulation is run up to $t^{*}=100$, and each symbol represents the mean of three simulations. Error bars denote the standard deviation, but are too small to be seen. Constant input simulations are marked by the open symbols, for $F^{*}=1$ and $T^{*}=2 / 3(\square), 0.80(\bigcirc), 0.87(+)$, $0.90(\times), 0.93(\diamond), 0.97(\nabla)$, and $1(\triangle)$. Effective rates associated with the modulated inputs of Fig. 4 are also simulated, with $\beta$ denoting the fraction of each period spent at $T_{\min }$.

we mean by "fast." The averaging theorem predicts that as the modulation frequency is raised, simulations that explicitly include modulation will approach those obtained using the constant effective transition rates. This point is illustrated in Fig. 12, which shows the evolution of model 1 under the conditions of Fig. 4, for various modulation frequencies.

In general, the modulation frequency required for effective rate behavior will depend on the transition mechanisms and their rates. To explore this fast limit, we isolate individual surface transition mechanisms, considering first adsorption with adatom desorption only, and then adsorption with adatom surface diffusion only. In both cases, simulations are run with a constant flux of $F^{*}=1$, and the temperature is modulated between $T^{*}=0$ and various finite tempera-

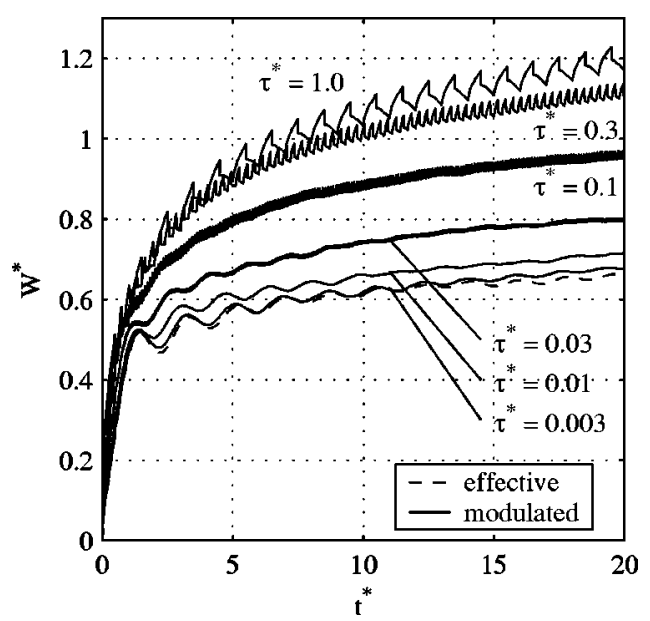

FIG. 12. Kinetic Monte Carlo simulations of model 1 on a $300 \times 300$ lattice, using the modulated inputs of Fig. 4. As the modulation period $\tau$ is decreased, the modulated simulations approach the predictions associated with the constant effective transition rates. 

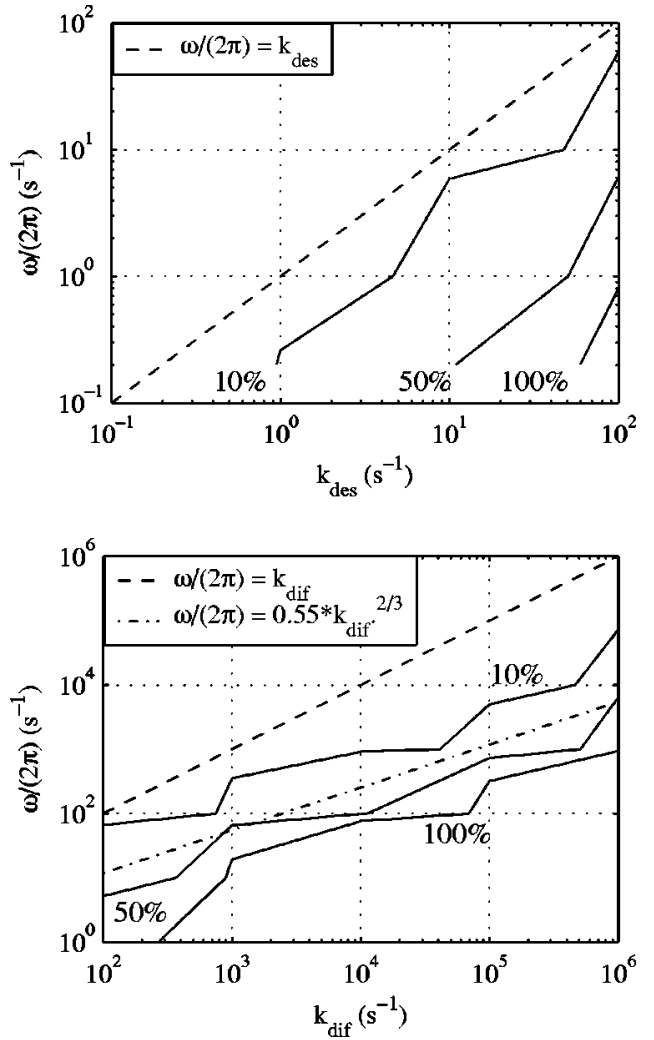

FIG. 13. Comparison between modulated growth and effective transition rates: (a) adsorption with adatom desorption; (b) adsorption with adatom surface diffusion. The solid lines represent error contours for the root-mean-square error in (a) height and roughness; (b) roughness and step density. Dashed lines are used to plot theoretical predictions.

tures over a range of modulation frequencies. The error between the modulated and effective rate simulations is then computed and represented by the contour plots of Fig. 13. The error in the first case, with adsorption and desorption only, is quantified as the root-mean-square error of the thickness and roughness, and is represented in Fig. 13(a). As the modulation frequency approaches the desorption rate, the modulated simulations approach the effective ones. In this case, the modulation must be faster than the rates of the surface transitions.

The simulations for adsorption and surface diffusion are summarized in Fig. 13(b), with error measured by surface roughness and step edge density. In these simulations, the error decays not according to the transition rate for surface diffusion, but instead sublinearly as the rate at which an adatom diffuses across an atomically flat terrace. This rate was computed using the parameter study of Evans and Bartelt, ${ }^{21}$ in which the characteristic length scale is equated to the interisland distance, and the island density $N$ is fitted as $N$ $=(0.55 \pm 0.02)\left(k_{\mathrm{dif}} / k_{\mathrm{ads}}\right)^{-0.3 \pm 0.03}$. The characteristic time is then the time required to hop a distance equal to the characteristic length via a random walk. Thus, to achieve the fast limit, the modulation frequency must be faster than characteristic time scales of surface evolution. However, in the case of surface diffusion, this does not require modulation faster than the fastest transition rate.

\section{B. Computation of periodic inputs}

We focus here on periodic inputs that switch between multiple process settings. In the examples, the input was modulated between two settings, but this may be generalized to $r$ settings. Defining $\alpha_{j}$ as the fraction of the period spent at input setting $\mathbf{u}_{j}$, one obtains $k_{\mathrm{eff}, i}=\sum_{j=1}^{r} \alpha_{j} k_{i}\left(\mathbf{u}_{j}\right)$. By definition, $\alpha_{j} \geqslant 0$ and $\sum_{j=1}^{r} \alpha_{j}=1$. This expression for the effective rates formally reduces to a problem in the theory of convex sets, in which the set of effective transition rates is the largest convex set containing the set of constantparameter transition rates. $^{22}$

The mathematical structure of the effective transition rates can be exploited to compute periodic process parameters. Defining $k_{\mathrm{obj}, i}$ as our objective transition rates, we may then define the following error function to be minimized: $\sum_{i=1}^{m}\left(k_{\mathrm{obj}, i}-\sum_{j=1}^{r} \alpha_{j} k_{i}\left(\mathbf{u}_{j}\right)\right)^{2}$, representing the error between the desired and the effective transition rates. One may then discretize $\mathbf{u}$ into $r$ representative settings, and compute the $\alpha_{j}$ to minimize the error. This optimization problem takes the specific form of a quadratic program. ${ }^{23}$ Furthermore, when the transition rates are all positive, the quadratic program always converges to global minimum of the error function. ${ }^{23}$ This optimization problem can be solved using MATLAB's quadratic programming function, and code has been included in Ref. 33.

To approximate the continuous range of process inputs, $r$ should be a large number, so the quadratic programming approach may produce complicated periodic inputs with $r$ settings. Caratheodory's theorem, a theorem in convex analysis, provides a way to simplify these complicated inputs. ${ }^{22}$ Specifically, the theorem states that the maximum number of points required to generate any convex combination is $m$ +1 , where $m$ is the dimension of the space. In this application, $m$ is the number of transition rates, so only a maximum of $m+1$ settings are required to construct any achievable convex combination. The proof of this theorem given also provides an algorithm to reduce the $r$ settings to $m+1 .^{22}$ The corresponding MATLAB code is found in our EPAPS deposit. $^{33}$

\section{DISCUSSION}

The averaging analysis developed in this paper provides a tool to simplify the interpretation of fast periodic inputs. The corresponding periodic process parameters may then be computed through quadratic programming. This overall approach requires that the desired effective transition rates be known, and that the modulation frequency be faster than the time scales of evolution. These two assumptions are discussed further in this section.

In general, the user will not know a priori which effective transitions rates will produce the desired surface properties. However, the averaging analysis simplifies the interpretation of periodic inputs by reducing the large number of independent parameters associated with the mean value and the modulation frequency, phase, and amplitude of each process input. This parameter set is replaced with the set of effective transition rates in the averaging analysis. However, the success of this approach does require that the user have some 
physical intuition regarding the effect of each constant transition rate on the final film properties, to guide the selection of desired effective transition rates. For example, one might understand based on physical intuition that the ratio of adsorption rate to diffusion rate should be minimized to achieve the smoothest surface. These effective rates must then be tested in simulation to verify the effect on surface properties.

A second key assumption in our analysis is that the modulation is fast relative to the time scales of growth, which may present practical challenges. However, throughout the last decade various film growth processes have been developed to deposit films under periodic process conditions. To modulate the flux of species to the surface, two primary methods have been employed: the use of valves to switch flow between the chamber and a vent line, ${ }^{24}$ and sequential exposure to flux as the substrate rotates through different environments. ${ }^{8}$ Methods for temperature modulation include resistive heating, ${ }^{25}$ laser irradiation, ${ }^{26}$ and supplemental cooling with water and liquid nitrogen. ${ }^{27}$ In electrodeposition, modulation is accomplished by pulsing the electrode current. $^{28}$ It may not be practical to speed up temperature modulation through backside resistive heating due to thermal inertia, or to speed up flux modulation through valve modulation due to transport delays. However, other methods, including the substrate rotation, ${ }^{8}$ laser excitation, ${ }^{26}$ and current modulation $^{28}$ are more anemable to fast modulation. The feasibility of achieving the fast limit of the averaging analysis must be assessed individually for each application, depending on the dominant time scales of surface evolution and the process inputs.

The analysis and algorithm presented in this paper apply only to fast modulation, but slower variation of the process, as such a temperature ramp, can also be beneficial. ${ }^{29}$ Other recent work by the authors describes a method for designing process parameters that vary more slowly, on the time scale of the overall process. ${ }^{30,32}$ This approach has also been applied to germanium homoepitaxy by Gallivan and Atwater. ${ }^{31}$
Thus, the averaging analysis presented here is not the only tool needed to compute process inputs, but instead describes one particular aspect. Further development of systematic mathematical approaches for process development is needed, but the initial work presented here suggests that this type of approach can provide a different insight.

\section{CONCLUSION}

In this paper we developed a method to analyze the effects of periodic process conditions when the period is short relative to the time scales of growth. The analysis is based on a lattice model of growth, in which the transition rates are strongly dependent on the process parameters. We showed that when the modulation of the process parameters is sufficiently fast, the film does not react to the individual pulses but instead evolves as if each periodic transition rate were replaced by a constant effective rate. Although the master equation for the lattice model is an extremely highdimensional differential equation, this method requires only an analysis of the transition rates.

The set of all possible effective rates is characterized in terms of the transition rates: this set is the smallest convex set containing the transition rates achievable with constant process parameters. With this mathematical framework we use a theorem of convex analysis to construct simple periodic process parameters. The periodic inputs are computed using quadratic programming, and when the desired effective rates are not achievable, then the inputs are computed that approximate the rates as closely as possible.

\section{ACKNOWLEDGMENTS}

This work was supported by DARPA/NSF under Grant No. DMS-9615858, by AFOSR under Grant No. F49620-951-0419, and by NSF. The authors would like to thank Harry Atwater for fruitful discussions.
*Electronic address: martha.gallivan@chbe.gatech.edu; URL: http://www.chbe.gatech.edu/gallivan

${ }^{1}$ M. E. Taylor and H. A. Atwater, Appl. Surf. Sci. 129, 159 (1998).

${ }^{2}$ G. Rosenfeld, N. N. Lipkin, W. Wulfhekel, J. Kliewer, K. Morgenstern, B. Poelsema, and G. Comsa, Appl. Phys. A: Mater. Sci. Process. 61, 455 (1995).

${ }^{3}$ G. Koster, G. J. H. M. Rijnders, D. H. A. Blank, and H. Rogalla, Appl. Phys. Lett. 74, 3729 (1999).

${ }^{4}$ S. Schinzer, M. Sokolowski, M. Biehl, and W. Kinzel, Phys. Rev. B 60, 2893 (1999).

${ }^{5}$ N. Combe and P. Jensen, Phys. Rev. B 57, 15553 (1998).

${ }^{6}$ V. A. Markov, O. P. Pchelyakov, L. V. Sokolov, S. I. Stenin, and S. Stoyanov, Surf. Sci. 250, 229 (1991).

${ }^{7}$ J. W. Klaus, S. J. Ferro, and S. M. George, J. Electrochem. Soc. 147, 1175 (2000).

${ }^{8}$ D. S. Olson, M. A. Kelly, S. Kapoor, and S. B. Hagstrom, J.
Mater. Res. 9, 1546 (1994).

${ }^{9}$ T. Sugiyama, T. Matsuura, and J. Murota, Appl. Surf. Sci. 112, 187 (1997).

${ }^{10}$ M. Svensson, U. Wahlstrom, and G. Holmbom, Surf. Coat. Technol. 105, 218 (1998).

${ }^{11}$ G. H. Gilmer and P. Bennema, J. Appl. Phys. 43, 1347 (1972).

${ }^{12}$ K. A. Fichthorn and W. H. Weinberg, J. Chem. Phys. 95, 1090 (1991).

${ }^{13}$ C. C. Battaile and D. J. Srolovitz, J. Appl. Phys. 82, 6293 (1997).

${ }^{14}$ A. B. Bortz, M. H. Kalos, and J. L. Lebowitz, J. Comput. Phys. 17, 10 (1975).

${ }^{15} \mathrm{~S}$. Wiggins, Introduction to Applied Nonlinear Dynamical Systems and Chaos (Springer-Verlag, New York, 1990).

${ }^{16}$ J. Baillieul, Int. J. Robust Nonlinear Control 5, 285 (1995).

${ }^{17}$ S. DasSarma and P. Tamborenea, Phys. Rev. Lett. 66, 325 (1991).

${ }^{18}$ A. Zangwill and E. Kaxiras, Surf. Sci. 326, L483 (1995).

${ }^{19}$ M. Tao, J. Appl. Phys. 87, 3554 (2000). 
${ }^{20}$ A. Robertson, T. H. Chiu, W. T. Tsang, and J. E. Cunningham, J. Appl. Phys. 64, 877 (1998).

${ }^{21}$ M. C. Bartelt and J. W. Evans, Phys. Rev. B 46, 12675 (1992).

${ }^{22}$ R. T. Rockafellar, Convex Analysis (Princeton University, Princeton, NJ, 1970).

${ }^{23}$ D. G. Luenberger, Linear and Nonlinear Programming (AddisonWesley, Reading, MA, 1984).

${ }^{24}$ S. Imai, S. Takagi, O. Sugiura, and M. Matsumura, Jpn. J. Appl. Phys., Part 1 30, 3646 (1991).

${ }^{25}$ D. W. Robinson and J. W. Rogers, Thin Solid Films 372, 10 (2000).

${ }^{26}$ Y. Suda, J. Vac. Sci. Technol. A 15, 2463 (1997).

${ }^{27}$ K. Eberl, W. Wegscheider, and G. Abstreiter, J. Cryst. Growth 111, 882 (1991).
${ }^{28}$ M. Schlesinger and M. Paunovic, Modern Electroplating (Wiley, New York, 2000).

${ }^{29}$ D. Yang, R. Jonnalagadda, B. R. Rogers, J. T. Hillman, R. F. Foster, and T. S. Cale, Thin Solid Films 332, 312 (1998).

${ }^{30}$ M. A. Gallivan, Ph.D. thesis, California Institute of Technology, 2002; see http://etd.caltech.edu/etd/available/etd-10222002$115711 /$

${ }^{31}$ M. A. Gallivan and H. A. Atwater, J. Appl. Phys. 95, 483 (2004).

${ }^{32}$ M. A. Gallivan and R. M. Murray, Int. J. Robust Nonlinear Control 14, 113 (2004).

${ }^{33}$ See EPAPS Document No. E-PRBMDO-69-002424 for the MATLAB code, which is used to compute the periodic process parameters (flux and temperature) to generate user-specified effective transition rates. 